\title{
Adaptive Segmentation Of EEG Signals
}

\section{Aufrichtigl, Richard; Pedersen, Simon Boel; Jennum, Poul}

\section{Published in:}

Proceedings of the Annual International Conference of the IEEE Engineering in Medicine and Biology Society

Publication date:

1991

Document Version

Publisher's PDF, also known as Version of record

Link back to DTU Orbit

Citation (APA):

Aufrichtigl, R., Pedersen, S. B., \& Jennum, P. (1991). Adaptive Segmentation Of EEG Signals. In Proceedings of the Annual International Conference of the IEEE Engineering in Medicine and Biology Society (Vol. Volume 13, pp. 453-454). IEEE.

\section{General rights}

Copyright and moral rights for the publications made accessible in the public portal are retained by the authors and/or other copyright owners and it is a condition of accessing publications that users recognise and abide by the legal requirements associated with these rights.

- Users may download and print one copy of any publication from the public portal for the purpose of private study or research.

- You may not further distribute the material or use it for any profit-making activity or commercial gain

- You may freely distribute the URL identifying the publication in the public portal

If you believe that this document breaches copyright please contact us providing details, and we will remove access to the work immediately and investigate your claim. 
Processing of Biological Signals 30.9-4

\title{
ADAPTIVE SEGMENTATION OF EEG SIGNALS
}

\author{
Richard Aufrichtig ${ }^{1,2}$, Simon Boel Pedersen ${ }^{1}$ and Poul Jennum ${ }^{3}$ \\ 'Electronics Institute, The Technical University of Denmark, DK 2800, Denmark \\ ${ }^{2}$ Dept. of Biomedical Engineering, Case Western Reserve University, Cleveland, OH 44106, USA \\ ${ }^{3}$ Dept. of Neurology, University Hospital of Copenhagen, Hvidovre Hospital, DK 2650, Denmark
}

\begin{abstract}
This paper decribes the application of adaptive segmentation techniques to EEG-signals. A number of methods to detect abrupt changes in the signal are discussed, assuming that it can be modelled as realizations of quasi-stationary autoregressive (AR) processes. Earlier published methods are revised to obtain symmetrical detection, so that a comparable segmentation performance is achieved independent of the change in power across a segment boundary. Simulation studies are used to rank the methods.
\end{abstract}

\section{INTRODUCTION}

EEG recordings vary due to changes in the physiological or psychological state of the subject. The implication is that the statistical characteristics of the EEG vary with time, and the signal is therefore considered to be non-stationary. This poses a significant problem in EEG analysis, since the basic assumption in the use of classical analysis methods (spectralperiod-and correlation-analysis) is that the signal is stationary.

The problem of detecting abrupt changes, and segmenting the signal into quasi-stationary parts has been a continuing subject of research for many years $[1,2,4,5]$. This study outlines the method of adaptive segmentation. It assesses some of the limitations of previous methods and presents modifications of them. The performance of the described algorithms are compared and evaluated in a simulation study.

\section{THEORY}

In order to perform a segmentation of the EEG signal we assume that a homogenous part of the signal can be modelled as a realization of an autoregressive (AR) process:

$$
x(n)=\sum_{k=1}^{p} a_{k} x(n-k)+e(n)
$$

where $p$ is the model order, ark are the AR-parameters and $e(n)$ is a zero mean white noise sequence with variance $\sigma_{\mathrm{E}}^{2}$.

The parametric AR-model has often been applied to EEG signals with orders varying from 5 to 15 depending on the length of the signal and the model application [1, 2]. The advantage of using an AR-model is that the statistical properties are solely described by the set of AR-parameters, which are constant within a stationary segment. It is therefore sufficient to detect a change in these parameters to detect a segment boundary.

The principle in adaptive filtering, as originally proposed by [4] is as follows. Features from an initial reference window are extracted and continuously compared with those of a moving window of EEG signal. A segment boundary is detected when the features of the reference window differ significantly from those of the moving window. A new reference window is then specified following the boundary and the procedure is repeated. Applying AR-modelling, the comparison between the reference and the moving windows can be done in the following ways:

I. An AR-model is estimated for the reference window and the signal in the moving window is filtered with the corresponding inverse filter. The basic assumption is that the output of the filtration will be a zero mean white noise residual signal. The comparison is therefore reduced to a test of how far the residual signal is from the white noise hypothesis. This method has been extensively used $[1,3,4,5]$ and a typical test statistic of how far the residual signal is from the white noise hypothesis has been based upon the cumulative sum of the residuals:

$$
z_{m}=\frac{1}{\sqrt{2 N_{m}}} \sum_{n=1}^{N_{m}}\left(\frac{e_{m}^{2}(n)}{\sigma_{r}^{2}}-1\right)
$$

where $N_{m}$ is the number of samples in the moving window, $e_{m}(n)$ is the residual signal and $\sigma_{\mathrm{r}}^{2}$ is the estimated noise variance of the reference window. Under the hypothesis that the signal is Gaussian and that no change occurs, this test statistic is asymptotically Gaussian distributed as $N(0,1)$. At first glance, the test seems to be effective, but it has been shown that it posses some serious drawbacks [3]. A problem occurs when the signal undergoes a change with a decrease in energy, i.e. when $\sigma_{\mathrm{m}}^{2}<\sigma_{\mathrm{r}}^{2}$. In this case the drift of the test statistic can be positive as well as negative. Such a situation occurs regularly in EEG signals, e.g. in sleep recordings with transitions between sleep stages.

II. A simple solution to the asymmetry of the test statistic in Eq. (2) can be achieved by performing a dual inverse filtering, followed by a calculation of a cumulative test statistic. More specifically, an AR-model is estimated for the reference window, followed by an inverse filtering of the moving window, and a calculation of the statistic $\mathrm{Z}_{\mathrm{m}}$. Now a new ARmodel is estimated for the moving window, followed by an inverse filtering and calculation of test statistic for the reference window. A fina statistic can now be calculated as the sum of the two intermediate values:

$$
\begin{aligned}
Z=Z_{m}+Z_{r} & =\frac{1}{\sqrt{2 N_{m}}} \sum_{n=1}^{N_{m}}\left(\frac{e_{m}^{2}(n)}{\sigma_{r}^{2}}-1\right) \\
& +\frac{1}{\sqrt{2 N_{r}}} \sum_{n=1}^{N}\left(\frac{e_{r}^{2}(n)}{\sigma_{m}^{2}}-1\right)
\end{aligned}
$$

This two step calculation insures a symmetrical detection of a change between two models, independent of whether there is an increase or decrease in energy across a segment boundary.

The use of Eq (3) is not without problems. Since it is now also necessary to estimate an AR-model for the moving window, the number of samples in this must be increased. The result is a smaller accuracy in the exact segment boundary. Furthermore the cumulated statistic does not follow a simple distribution due to the dependency between the two inverse filtrations. This means, that the segment boundary must be determined, when $Z$ is larger than an empirically calculated threshold value. In practice this does not pose a problem, since the EEG signal is rarely Gaussian distributed. Empirical thresholding is therefore generally also required when Eq. (2) is used.

III. A different approach from the inverse filtering, which can be used to compare the AR-parameters of the reference and moving windows has been described in [6]. In the derivation of the method, the asymptotic Gaussian 
distribution of the AR-parameters is used to achieve a test statistic for the difference between the two sets of AR-parameters:

$$
z_{1}=\frac{N_{r} N_{m}}{\sigma_{r}^{2}\left(N_{r}+N_{m}\right)}\left[\left(a_{r}-a_{m}\right)^{T} R_{p, r}+\frac{\left(\sigma_{r}^{2}-\sigma_{m}^{2}\right)^{2}}{2 \sigma_{r}^{2}}\right]
$$

where $\mathrm{N}$ are the number of samples, $\mathrm{a}=\left[\mathrm{a}_{1}, \mathrm{a}_{2}, \ldots \mathrm{a}_{\mathrm{p}}\right]^{\mathrm{T}}$ are the estimated AR-coefficients, $\sigma^{2}$ is the estimated noise variance and $R_{p}$ is the $p \times p$ auto-correlation matrix of the signal. The subscripts $r$ and $m$ refer to the reference and moving windows respectively. It is shown that $Z_{1}$ is asymptotically $\chi^{2}(p+1)$ distributed.

IV. It can be seen directly from Eq. (4) that since only $\mathbf{R}_{\mathrm{r}}$ is used, this test also possesses some asymmetry, and to compensate for this, the following statistic is constructed:

$$
\mathrm{Z}_{12}=\mathrm{Z}_{1}+\mathrm{Z}_{2}
$$

where $Z_{1}$ is identical to the expression in Eq. (4) while $Z_{2}$ has the subscripts $r$ and $m$ reversed, when compared to Eq. (4).

Compared to $Z_{1}$ in Eq. (4) there is only a very little increase in the computational work when $Z_{12}$ is used, since both auto-correlation matrices already have been calculated during the parameter estimation. As in the case of the dual inverse filtration test, the test statistic, $Z_{12}$, must be compared to an empirical threshold value.

\section{EVALUATION OF METHODS}

Methods I, II, III and IV described in the previous section has been applied to simulated as well as real EEG's [1]. An example of a simulated test signal is shown in Figure 1.

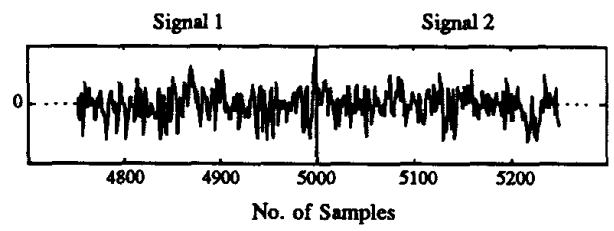

Figure 1. Part of test signal at boundary between simulated realizations of two AR(14)-models.

The test signal consist of $2 \times 5000$ samples, from two simulated realizations of AR(14)-models. The first part (Signal 1) is a simulated EEG signal from 14 AR-parameters estimated from a true EEG signal. For the simulation of the second part (Signal 2), one of the reflection coefficients from the model of Signal 1 was changed (there is a direct relationship between the AR-parameters and the reflection coefficients of an AR-model. The advantage of changing a reflection coefficient, to obtain an new model, is that stability of the system is insured, as long as the absolute values of the reflection coefficients are less than one [1]).

The variance of the noise was $\sigma_{\text {el }}^{2}=0.35$ for Signal 1, while it was changed to $\sigma_{e 2}^{2}=0.48$ for Signal 2. Since a transition from Signal 1 to Signal 2 is an increase in $\sigma_{e}^{2}$, one would therefore expect all 4 methods to detect the change, while at least method I would fail, if the two signal were reversed. This is confirmed by the result in Figure 2. Notice that the reference window is kept at the initial position, even after the transition, to follow the values of the test statistics. A significant improvement is achieved when either method II or IV is used, and it is in both cases possible to detect the boundary (absolute symmetry is not achieved, since the reference windows are different in the two cases). No significant difference has been observed between method II and IV, and method IV should therefore be recommended, since it is purely parametrical and does not require inverse filtering.

\section{REFERENCES}

[1] R. Aufrichtig, "Adaptive segmentation of EEG signals", Master Thesis, Institute of Electronics, The Technical University of Denmark, (in Danish), Denmark: 1990.

[2] J.S. Barlow, "Methods of Analysis of Nonstationary EEGs, with Emphasis on Segmentation Techniques: A Comparative Review", J. Clin. Neurophysiol., Vol. 2, pp. 267-303: 1985.

[3] M. Basseville and A. Benvenista, "Sequential Segmentation of Nonstationary Digital Signal Using Spectral Analysis", Information Sciences, Vol. 29, pp. 57-73: 1983.

[4] G. Bodenstein and H.M. Praetorius, "Feature Extraction from the Electroencephalogram by Adaptive Segmentation", Proc. IEEE, Vol. 65, pp. 642-652: 1977.

[5] A.C. Sanderson and J. Segen, " Hierarchical Modelling of EEG Signals", IEEE Trans. Pat. Anal. Mach. Intel., Vol. PAMI-2, pp. 405-415: 1980

[6] P. Stoica, "Performance Evaluation of some Methods for Off-Line Detection of Changes in Autoregressive Signals". Signal Process. Vol. 19, pp. 301-310: 1990.

Address for correspondence:

Richard Aufrichtig

Department of Biomedical Engineering

Wickenden Building

Case Western Reserve University

Cleveland, $\mathrm{OH} 44106$

Tel: $216-3688812$
Figure 2.

$\checkmark$ alues of test statistics after segmentation in test signal. In the right column $(A, B, C$ and D) the reference window was the first $\mathbf{3 0 0}$ samples of Signal 1 , while the moving window (also 300 samples) was stiding along signal 1 followed by signal 2. In the left column (E, $F, G$ and $H$ ) the signals were reversed. Now the reference window was the first 300 samples of Signal 2, while the moving window was sliding along the rest of Signal 2 followed by Signal 1 .

Fig. $A$ and $E$ : method I

Fig. B and F: method II

Fig. $C$ and G: method II

Fig. D and $\mathrm{H}$ : method IV
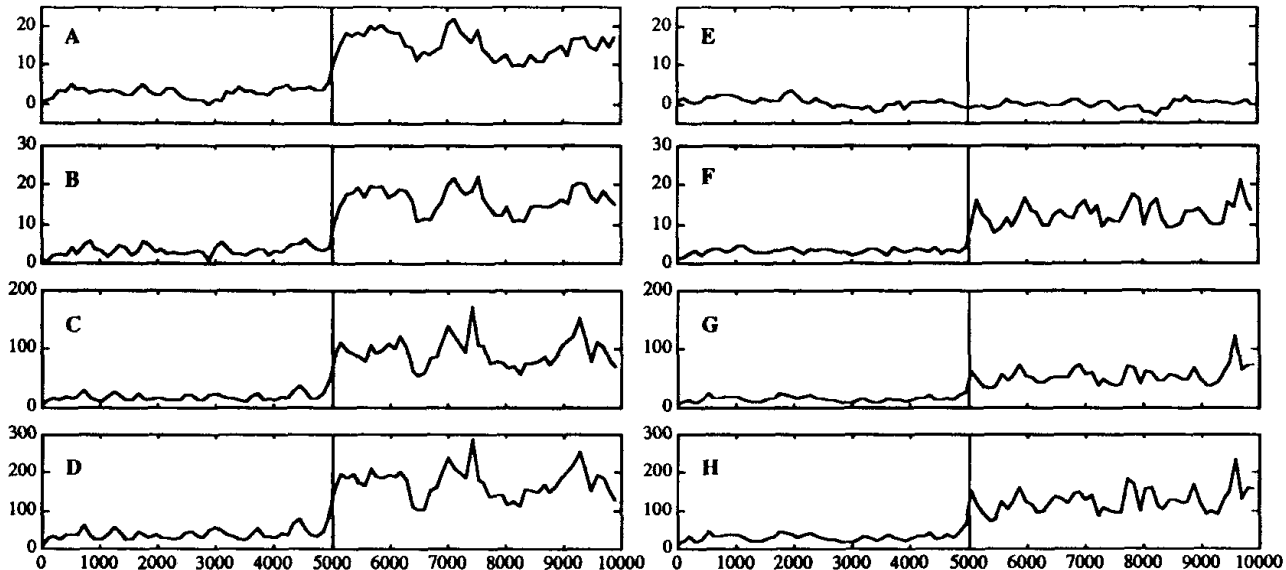